\title{
Estudo das abreviaturas dos documentos de Capivari do século XIX
}

\author{
Rosicleide Rodrigues Garcia*
}

RESUMO: Este artigo, pertencente à dissertação Formação e expansão do dialeto caipira na cidade de Capivari, sob orientação do Prof. Dr. Manoel Mourivaldo Santiago Almeida, da Universidade de São Paulo, ${ }^{1}$ utilizou o labor filológico para editar 72 documentos cartoriais do século XIX da cidade de Capivari a fim de buscar a fidedignidade dos fólios e auxiliar futuras pesquisas acerca da identificação e desenvolvimento de abreviaturas século XIX. Os estudos paleográficos valeram-se, além da análise caligráfica, do levantamento exaustivo de abreviações nos documentos e, por fim, sua reprodução fac-similar.

PALAVRAS-CHAVE: Edição de fólios; paleografia, abreviaturas novecentistas; filologia.

\section{Introdução}

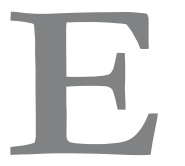

ste trabalho faz parte de um dos capítulos da dissertação de mestrado Formação e expansão do dialeto caipira em Capivari. Situada a 108 quilômetros de São Paulo e fundada em 1832, a cidade fora berço do imortal Amadeu Amaral, autor da obra O dialeto caipira (1922), primeiro estudo a preocupar-se com a diversidade do falar paulista, e, tendo-as como elementos de estudo, fora realizada uma busca exaustiva de variantes linguísticas em documentos capivarianos cartoriais do século XIX, de modo a expressar (ou não) os estudos dialetais feitos pelo autor em questão, demonstrando o que já pertencia à língua antes de suas observações.

\footnotetext{
Mestranda do Programa de Pós-Graduação em Filologia e Língua Portuguesa da Universidade de São Paulo - rhozzi@gmail.com.

1 Este é um subprojeto do Projeto Caipira, que está desenvolvendo pesquisas relacionadas à comunidade lingüística de São Paulo por pessoal ligado basicamente à área de Filologia e Língua Portuguesa da USP.
} 
Para alcançar esses objetivos, a tese se vale do labor filológico. Para isso, fora realizado o estudo paleográfico dos documentos e um levantamento exaustivo de abreviações presentes nos fólios transcritos a fim de contribuir com futuras pesquisas acerca do assunto, considerando que no Brasil há pouco escrito a respeito, principalmente quando se trata de século XIX. Ressaltase que estudos individualizados de suporte, como composição do papel, história da empresa fabricante, entre outros, não foram realizados por não pertencerem ao objetivo da dissertação.

Assim, este artigo trará parte do capítulo que antecede as edições, explicando como fora feito o processo recensio e um quadro de abreviaturas facsimiladas.

\section{O processo de Recensio}

A documentação sobre a cidade de Capivari é constituída basicamente por documentos dispositivos, testemunhais e informativos ascendentes e horizontais. ${ }^{2}$

Para a constituição do corpus da dissertação, foram escolhidas escrituras, atas, abaixo-assinado, faturas, posturas, processos, propostas, censos e requisições emanados em ofícios ${ }^{3}$ da cidade, provenientes do período colonial. Os primeiros fólios - 45r a 46v - são do Livro de Notas n 2 de 1785 do Museu e Arquivo Municipal de Itu (MAHMI), em que está registrado o lançamento da sesmaria do capitão André Sampaio Botelho, responsável pela formação da cidade que se ergueria posteriormente; os segundos são os fólios 63r e 63v do Livro de Notas n 19 de 1808 do Museu Republicano "Convenção de Itu", sendo uma escritura de compra e venda da cita cidade de um terreno no Bairro de Capivari; os documentos que seguem são fólios diversos das caixas nº 54, 185, 186 e 187, séries CO0234, CO0980, CO0981 e CO0982

2 Segundo Belloto (2002, p.28-29), diz-se que os dispositivos são documentos normativos, que emanam do Poder Legislativo, Executivo ou por autoridades administrativas; de ajuste, que são pactuais, podendo ser tratado, termo, contrato; e correspondências derivadas dos atos normativos. Os testemunhais são os ocorridos depois do cumprimento de um ato dispositivo, como atas, assentamentos, certidões, entre outros. Os informativos são os que esclarecem questões contidas em outros documentos, sendo eles relatórios, pareceres, informações etc. Ainda dentro das definições da autora (2002, p.37), os ascendentes são aqueles em que os súditos se comunicam com seus superiores, e horizontais são os documentos distribuídos entre pessoas/autoridades do mesmo nível.

3 As classificações documentais também seguem as definições prescritas por Belloto (São Paulo, 2002). 
(respectivamente) de 1819 a 1888 do Arquivo do Estado de São Paulo, sendo o de 1819 um ofício escrito pelo padre fundador da primeira capela do território estudado, e os de 1821 e 1829 feitos por habitante próprio da região. A partir de 1832 os ofícios são relativos aos acontecimentos da câmara e constituição de Capivari, editados ano após ano, ressaltando que todos são de origem brasileira e paulista.

\title{
2. Aspectos paleográficos dos manuscritos
}

Como a documentação é basicamente formada por documentação oficial, ela segue uma característica formal, com cuidado com a forma e apresentação. Assim como vemos em Acioli (1994, p. 55), em sua maioria os documentos apresentam-se

caligrafados, [...] no tipo cursivo e sobre papel, sem traçado de linhas. As páginas manuscritas têm regras de diversas larguras mas são delimitadas muito uniformemente, dando uma fisionomia agradável à composição. [...] É habitual redigi-las quase em coluna, na segunda metade da folha, deixando-se a primeira, à esquerda.

O instrumento utilizado para a escrita foi a pena de ave. Aproximadamente $98 \%$ dos manuscritos foram redigidos com tinta de cor castanha, sendo apenas em 2\% usada a tinta azul. As letras serão denominadas nacionais novecentistas cursivas - por se tratar de documentos brasileiros e possuidores de uma característica própria no século XIX “, mostrando, em grande parte, um traçado leve, de módulos estreitos, de ductus regulares, inclinadas para a direita e espaçadas na linha, confundindo, às vezes, a delimitação da fronteira de palavras. Em sua maioria, os ofícios foram escritos por mãos hábeis, havendo pouquíssimos casos de presença de inábeis.

Quanto às assinaturas e autenticidade documental, novamente vemos em Acioli (1994, p. 57) que

\begin{abstract}
geralmente redigidos por escrivões de notas, os documentos têm nas assinaturas a definição de sua autenticidade ou falsidade. [...] Os autógrafos vêm, às vezes, acompanhados por cetras (laçaria caligráfica acrescentada a uma firma que dificultam a sua interpretação). Também é grande o número de nomes e sobrenomes abreviados e não existe uma rigidez na composição das letras que formam a palavra.
\end{abstract}

Os sinais alógrafos são raros, aparecendo em quantidade razoável apenas nos fólios do XVIII e no início do XIX, mas em todo o período estudado elas se restringem a uma padronização e similaridade. 
176 GARCIA, Rosicleide Rodrigues. Estudo das abreviaturas dos documentos de Capivari do século XIX

\section{As abreviaturas}

De acordo com Costa, em seu estudo publicado na Revista Histórica Online do Arquivo do Estado de São Paulo, as abreviaturas encontradas nos manuscritos podem dividir-se em:

- Apócope ou suspensão: supressão de elementos finais da palavra;

- Sigla: consiste na representação da palavra pela letra inicial maiúscula, seguida de ponto. Pode ser simples ou reduplicada;

- Síncope ou contração: representa a supressão de letras do meio do vocábulo;

- Sobrescritas: sobreposição da última ou das últimas letras da palavra;

- Mista: quando em uma mesma palavra se encontram abreviaturas por suspensão (apócope) e por contração (síncope), ou quando, numa seqüência de palavras, nenhuma delas apresenta-se isoladamente abreviada;

- Sinal especial: presença de um sinal colocado no início, meio ou fim da palavra abreviada, indicando os elementos ausentes;

- Numéricas: constituem as abreviaturas de numerações, designativas de ordem, divisão e meses do ano. Utiliza-se a sobreposição das letras $o$ e $a$ minúsculas aos numerais ou à terminação - $b r$.

- Notação tironiana: sinal criado por Tiro, escravo de Cícero.

Desse modo, fora feito um quadro elucidativo de forma que possamos vislumbrar e compreender quais abreviaturas foram mais utilizadas durante o século XIX segundo os documentos selecionados.

\subsection{Quadro de abreviaturas dos documentos de Capivari}

\section{Aро́соре оu suspensão}

\begin{tabular}{|c|c|l|l|}
\hline Abreviatura & Desenvolvimento & Ano & Fac-símile \\
\hline Anim & animal & 1836 & 1885 \\
\hline Arr. & Arruda & $1851,1878,1886$ \\
\hline Art. & Artigo & 1886 & \\
\hline Cit. & citada & 1851 & Cons \\
\hline Cod. & Codigo & 1886 & \\
\hline Const. & Constituinte & & \\
\hline
\end{tabular}




\begin{tabular}{|c|c|c|c|}
\hline Doc. & documento & 1884,1886 & 20 \\
\hline Loc. & locuçaõ & 1886 & \\
\hline Nov. & Novembro & 1882 & $\alpha$. \\
\hline Ord. & ordem & 1884 & \\
\hline Pen. & penal & 1851 & \\
\hline
\end{tabular}

\section{Especial}

\begin{tabular}{|c|l|l|l|}
\hline Abreviatura & Desenvolvimento & \multicolumn{1}{|c|}{ Ano } & Fac-símile \\
\hline$@$ & arrobas & $\begin{array}{c}1841, \quad 1864 \mathrm{c}, \\
1882\end{array}$ & 1835 \\
\hline$E t a$ & et cetera & 1843 & \\
\hline$e t t$ & et cetera & 1843 & $\S$ \\
\hline$t t$ & et cetera & $1880,1884,1886$ & \\
\hline \multirow{2}{*}{$s$} & paragrafo & & \\
\hline
\end{tabular}

\section{Mista}

\begin{tabular}{|c|c|c|c|}
\hline Abreviatura & Desenvolvimento & Ano & Fac-símile \\
\hline E.R.M $M^{c e}$ & $\begin{array}{l}\text { Espera Receber } \\
\text { Merce }\end{array}$ & 1888 & $\mathscr{B} \mathscr{B} \subset \mathbb{C}^{*}$ \\
\hline E.E.R.M $M^{c e}$ & $\begin{array}{l}\text { E Então Recebera } \\
\text { Merce }\end{array}$ & 1886 & $9 f^{e a}$ \\
\hline S. $E x^{a}$ & Sua Excellencia & 1836, 1855 & $x \cdot 2$ \\
\hline$V . S^{a}$ & Vossa Senhoria & 1838, 1855, 1879 & 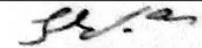 \\
\hline V.V.S.S. ${ }^{a s}$ & Vossas Senhorias & 1888 & gar \\
\hline V.Ex $x^{a}$ & Vossa Excellencia & 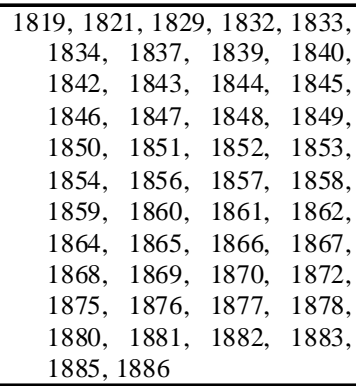 & \\
\hline V.Ex $x^{c a}$ & Vossa Excellencia & 1851,1884 & ley.ea \\
\hline
\end{tabular}


178 GARCIA, Rosicleide Rodrigues. Estudo das abreviaturas dos documentos de Capivari do século XIX

Numérica

\begin{tabular}{|c|c|c|c|}
\hline Abreviatura & Desenvolvimento & Ano & Fac-símile \\
\hline $1^{\circ}$ & primeiro & 1882 & \\
\hline $10 b r^{\circ}$ & dezembro & 1855 & \\
\hline $10^{\text {bro }}$ & dezembro & 1874 & \\
\hline $7 b r^{\circ}$ & setembro & $\begin{array}{l}1836,1839,1840,1841,1864 \\
1872,1880\end{array}$ & \\
\hline $8 b r^{\circ}$ & outubro / oitubro & $\begin{array}{l}\text { 1838, 1839, 1841, 1851, 1854, } \\
\quad 1858,1864,1878\end{array}$ & \\
\hline $9 b r^{\circ}$ & novembro & 1840, 1846, 1862, 1873 & \\
\hline$p^{r 100}$ & porcento & 1854 & \\
\hline
\end{tabular}

\section{Sigla}

\begin{tabular}{|c|c|c|c|}
\hline Abreviatura & Desenvolvimento & Ano & Fac-símile \\
\hline$C M$ & Camara Municipal & 1841 & \\
\hline$D$ & Dona & 1821, 1841, 1843, 1884 & \\
\hline$D$ & Dignissimo & $\begin{array}{l}1858,1859,1860,1861,1862, \\
1870\end{array}$ & \\
\hline$D V$ & $\begin{array}{l}\text { Documento de } \\
\text { Venda }\end{array}$ & 1808 & \\
\hline G. & guarda & 1836 & \\
\hline$J$ & Jose & 1887 & \\
\hline$L$ & Livro & 1884 & \\
\hline$M$ & Magalha?s & 1887 & \\
\hline$M$ & Municipal & 1838 & \\
\hline$M$. & Mui & 1870 & \\
\hline M. $d$. & Mui dignissimo & $\begin{array}{l}\text { 1855, 1873, 1874, 1877, 1880, } \\
\text { 1881, 1882, 1883, 1884, } \\
\text { 1885, 1887 }\end{array}$ & \\
\hline
\end{tabular}




\begin{tabular}{|c|c|c|c|}
\hline$P$ & Prezidente & 1874 & \\
\hline$P$ & Provincia & 1866 & \\
\hline$P$ & proximo & 1869 & \\
\hline$P P$ & proximo passado & $\begin{array}{l}\text { 1836, } 1839,1848,1852,1853 \\
\quad 1874\end{array}$ & \\
\hline$P P$ & Pedimos Permissão & 1886 & \\
\hline$S$ & Saõ / Sam & 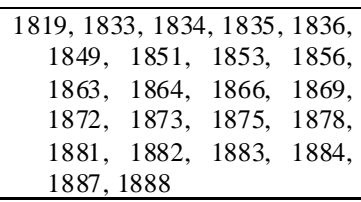 & \\
\hline$T$ & Teixeira & 1886 & \\
\hline$S$. & Santo (a) & 1835,1836 & \\
\hline$S$. & Sua & 1836 & \\
\hline S.S. & Sua Senhoria & 1863 & \\
\hline V.V.S.S & Vossas Senhorias & 1841 & \\
\hline$V g$ & verbi gratia & 1847 & \\
\hline$V P$ & $\begin{array}{l}\text { Vice Prezidente / } \\
\text { Vice Presidente }\end{array}$ & 1844,1851 & \\
\hline VS & Vossa Senhoria & 1860 & \\
\hline
\end{tabular}




\section{Síncope ou contração}

\begin{tabular}{|c|c|c|c|}
\hline Abreviatura & Desenvolvimento & Ano & Fac-símile \\
\hline etr & et cetera & 1847 & \\
\hline fs & folhas & 1886 & \\
\hline Glz & Gonçalvez & 1821 & \\
\hline GO & Gonçallo & 1861,1864 & \\
\hline Illmo & Illustris simo & 1861,1878 & \\
\hline$L b$ & libra & 1854 & \\
\hline Miz & Martinz & $\begin{array}{l}\text { 1835, 1836, 1838, 1840, 1845, } \\
\begin{array}{l}\text { 1846, 1847, 1863, 1864, } \\
1878\end{array}\end{array}$ & \\
\hline$M l$ & Manoel & 1878 & \\
\hline$P g$ & pagina & 1886 & \\
\hline$P g$ & pago & 1860 & \\
\hline Roiz & Rodriguez & 1836, 1856, 1858, 1860 & \\
\hline Qxen & quando & 1880 & \\
\hline Senr & Senhor & $\begin{array}{l}1861,1865,1872,1873,1874, \\
1875,1877,1880\end{array}$ & \\
\hline Snr & Senhor & 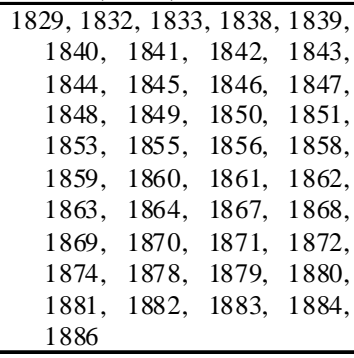 & \\
\hline Tbem & tambem & 1840,1854 & \\
\hline Tbam & tabeliam & 1877 & \\
\hline
\end{tabular}




\section{Sobrepostas}

\begin{tabular}{|c|c|c|c|}
\hline Abreviatura & Desenvolvimento & Ano & Fac-símile \\
\hline acintosam $^{e}$ & acintosamente & 1855 & exivan...e \\
\hline acolhim $^{\text {to }}$ & acolhimento & 1854 & \\
\hline actualm $^{e}$ & actualmente & 1833, 1836, 1838, 1843, 1861 & acteralsoe \\
\hline administr $^{m}$ & administraçam & 1861 & ancen \\
\hline$A g^{a r}$ & Aguiar & 1863 & \\
\hline$A g^{t o}$ & Agosto & 1871 & \\
\hline $\operatorname{Alg}^{a}$ & alguma & 1854 & \\
\hline $\operatorname{alg}^{a s}$ & algumas & 1854 & \\
\hline $\operatorname{alg}^{m a}$ & alguma & 1874 & \\
\hline $\operatorname{alg}^{m}$ & algum & 1855 & \\
\hline$a l g^{s}$ & alguns & 1854 & \\
\hline$A l m^{d a}$ & Almada & 1878 & \\
\hline $\operatorname{Almd}^{a}$ & Almeida & $\begin{array}{c}1833,1836,1841,1851,1852 \\
1858,1859,1863,1864\end{array}$ & \\
\hline Alt. & Altissimo & 1870 & \\
\hline$A m^{a l}$ & Amaral & $1858,1859,1861$ & comis \\
\hline$B r r^{s}$ & Barros & 1860 & \\
\hline $\mathrm{Cam}^{a}$ & Camara & $\begin{array}{l}\text { 1836, 1842, 1845, 1849, 1851, } \\
1859,1861\end{array}$ & \\
\hline $\mathrm{Camg}^{\circ}$ & Camargo & $1838,1840,1847$ & \\
\hline $\mathrm{Cam}^{\circ}$ & Camargo & 1836 & en \\
\hline $\mathrm{cam}^{\circ}$ & caminho & 1836, 1838 & Cam: \\
\hline cam $^{o s}$ & caminhos & 1835 & Ba \\
\hline Camr $^{a}$ & Camara & 1844 & $\ldots a^{\infty}$ \\
\hline $\operatorname{Cap}^{a l}$ & capital & 1860 & \\
\hline
\end{tabular}




\begin{tabular}{|c|c|c|c|}
\hline $\operatorname{Cap}^{m}$ & Capitam & 1836, 1838, 1840, 1843 & \\
\hline $\operatorname{Card}^{o}$ & Cardoso & 1836 & \\
\hline$c r^{\circ}$ & criado & 1854,1870 & $C_{x}$. \\
\hline$d^{a}$ & dita & 1838, 1854 & \\
\hline$d a q^{\text {lles }}$ & daquelles & 1844 & \\
\hline$d^{a s}$ & ditas & 1829 & \\
\hline defenitivam $^{e}$ & defenitivamente & 1833 & Defescutrasen" \\
\hline$d e n r^{\circ}$ & denheiro & 1857 & Se \\
\hline denovam $^{e}$ & denovamente & 1857 & $2 e$ \\
\hline $\operatorname{Des}^{\circ}$ & despenso & 1854 & \\
\hline $\operatorname{Desp}^{\circ}$ & despacho & 1863 & \\
\hline$d e p^{s}$ & depois & 1854 & \\
\hline $\operatorname{dezbr} r^{o}$ & dezembro & 1829, 1862 & \\
\hline$D e z^{o r}$ & Dezembargador & 1843 & \\
\hline$d^{o}$ & dito / ditto & $\begin{array}{l}\text { 1821, 1832, 1838, 1843, 1844, } \\
\quad 1854,1862,1863\end{array}$ & \\
\hline$D^{o r}$ & Doutor & 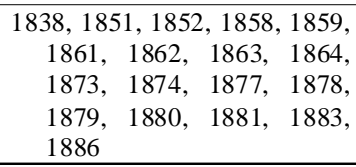 & \\
\hline$D^{r}$ & Doutor & $\begin{array}{l}1854,1864,1865,1870,1871, \\
1872,1874,1875,1876, \\
1884\end{array}$ & \\
\hline$d r^{\circ}$ & dinheiro & 1849 & \\
\hline$d^{r o}$ & dinheiro & 1863 & 0 \\
\hline$D^{e s}$ & Deus & 1880 & \\
\hline
\end{tabular}




\begin{tabular}{|c|c|c|c|}
\hline$D^{s}$ & Deos / Deus & 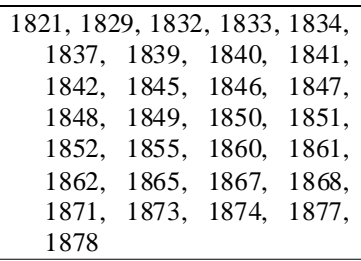 & \\
\hline edonied $^{e}$ & edoniedade & 1836 & comith \\
\hline emidiatam $^{\text {te }}$ & emidiatamente & 1829 & earidin=temo \\
\hline$e n t t^{\circ}$ & entanto & 1854 & \\
\hline entirinam $^{e}$ & entirinamente & 1833 & intivinaso" \\
\hline$E s c r^{A M}$ & Escrivam & 1836 & \\
\hline$e s c r^{\circ}$ & escravo & 1821 & \\
\hline escros & escravos & 1836 & \\
\hline$G c^{i a}$ & Garcia & 1886 & \\
\hline$g^{e}$ & guarde & 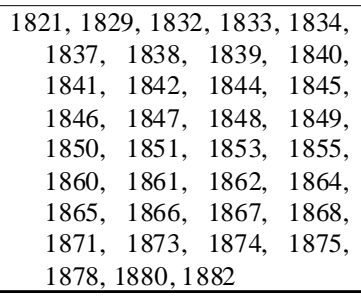 & \\
\hline $\operatorname{Gov}^{\circ}$ & Governo & 1836, 1838, 1839, 1840, 1861 & \\
\hline Guim $^{\text {es }}$ & Guimarães & 1836 & \\
\hline houver $^{m}$ & houverem & 1843 & he \\
\hline Ianr $^{\circ}$ & Ianeiro & $1833,1842,1849$ & Cont \\
\hline$I^{e}$ & Ioze & 1836 & \\
\hline $\operatorname{Ign}^{e o}$ & Ignaceo & 1836 & \\
\hline igoald $^{e}$ & igoaldade & 1841 & 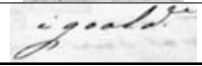 \\
\hline
\end{tabular}


184 GARCIA, Rosicleide Rodrigues. Estudo das abreviaturas dos documentos de Capivari do século XIX

\begin{tabular}{|c|c|c|c|}
\hline$I l^{m o}$ & & Illustrissimo & Yll. mo \\
\hline$m^{a}$ & minha & $\begin{array}{l}\text { 1821, 1832, 1843, 1849, 1854, } \\
\quad 1855,1864\end{array}$ & $\operatorname{ma}$ \\
\hline mad $^{\text {ras }}$ & madeiras & 1854 & $\mathrm{Ca} ;$ \\
\hline $\mathrm{Mag}^{\mathrm{s}}$ & Magalha?s & 1883,1884 & \\
\hline$M^{a l}$ & Municipal & 1851, 1855, 1872, 1878 & 2 \\
\hline Mun $^{\circ}$ & Municipio & 1861 & etberes". \\
\hline$M^{e l}$ & Manoel & 1834, 1882 & \\
\hline$m a n r^{a}$ & maneira & 1835 & $\approx$ \\
\hline$m^{m o}$ & mesmo & $\begin{array}{l}\text { 1836, 1839, 1841, 1842, } 1850 \\
\begin{array}{l}1854,1855,1861,1863, \\
1865\end{array}\end{array}$ & sen. \\
\hline perezid $^{e}$ & perezidente & 1855 & Dresich \\
\hline$p^{l o}$ & pelo & 1854, 1863 & \\
\hline prem $^{\text {ro }}$ & premeiro & 1854 & \\
\hline Pres $^{e}$ & Presidente & 1875 & \\
\hline$q^{r}$ & quer & 1835, 1836, 1842, 1843, 1854 & \\
\hline$q^{r o}$ & quero & 1854 & \\
\hline$Q^{r o ́ s}$ & Queirós & 1863 & \\
\hline$q^{t_{a}}$ & quantia & 1840 & \\
\hline$T t^{a}$ & testemunha & $1836,1843,1854$ & \\
\hline$t t^{a s}$ & tantas & 1861 & \\
\hline$t t^{\circ}$ & titulo & 1841, 1854 & \\
\hline$t t^{\circ}$ & tanto & 1854 & \\
\hline$t e^{o s}$ & testemunhos & 1843 & \\
\hline
\end{tabular}




\begin{tabular}{|c|c|c|c|}
\hline$V^{a}$ & \multicolumn{1}{|c|}{ villa } & $\begin{array}{c}1833,1834,1836,1837,1838, \\
1839,1840,1841,1843, \\
1844,1845,1846,1847, \\
1848,1849,1860,1861\end{array}$ \\
\hline$V^{a} \cdot E^{a}$ & Vossa Excellencia & 1873,1880 & \\
\hline$V^{a} . E x^{a}$ & Vossa Excellencia & 1838,1886 & \\
\hline$v^{\text {or }}$ & venerador & 1854,1870 & \\
\hline$V^{\circ} g^{\circ}$ & Vigario & 1847 & \\
\hline$v^{2} t^{e}$ & vontade & 1854,1861 & \\
\hline$X^{\text {er }}$ & Xavier & 1874,1875 & \\
\hline
\end{tabular}

\section{Notação tironiana}

\begin{tabular}{|c|c|c|}
\hline & $1819,1821,1829,1832,1833$, \\
& $1834,1835,1836,1837$, \\
$1838,1839,1840,1841$, \\
$1842,1843,1844,1845$, \\
$1847,1848,1850,1853$, \\
$1854,1855,1857,1860$, \\
\end{tabular}

\section{Conclusão}

As edições são amplamente esclarecedoras. Nelas vimos que o uso de abreviaturas fora mais constante do início aos meados do século XIX, rareando-se a partir de 1870, permanecendo mais freqüentemente aquelas que se tornaram convenções, como $I l l^{\text {mo }}, E x^{\text {mo }}, V$. Ex. ${ }^{a}$ e as siglas. Nota-se que as sobrepostas, que foram utilizadas largamente para a abreviação de advérbios de modo, não são administradas no período citado, não importando o tamanho do documento (a considerar que antes elas eram mais presentes em textos longos). Pontuamos, ademais, que os fólios dos livros de notas não as apresentaram em seu conteúdo, o que nos leva a crer que já se havia uma consciência de uso e, considerando as aparições que uma determinada abreviatura tinha em certos documentos, eram produzidos dependendo do hábito de seus escrivães, como exemplo o ' $q$ ' constantemente presente nos fólios dos anos de 1854 e 1841. 
Outro ponto a ser observado é a ausência em bibliografias de uma denominação para as grafias novecentistas, por isso a adoção de nacionais novecentistas cursivas para classificação geral, pois se tratam de uma escrita característica e brasileira, embora em muitos casos pareçam coincidentes com a caligrafia oitocentista, elas apresentam diferenças quanto ao uso de sinais gráficos e curvatura de linhas (por vezes mais estreitas, outras arredondas, entre outros).

Assim, embora esclarecedoras para a compreensão visual, as edições ainda deixam muitas questões em aberto que necessitam ser verificadas com mais empenho, o que, infelizmente, não é o objetivo deste trabalho, mas, como qualquer contribuição filológica, tem a obrigação de clarificar para os demais pesquisadores da área.

\section{Bibliografia}

ACIOLI, V. L. C. (1994) A escrita no Brasil colônia: um guia para leitura de documentos manuscritos. Recife: Editora Universitária.

BASSETO, B. F. (2001) Elementos de filologia românica. São Paulo: Edusp.

BELLOTO, H. L. (2002) Como fazer análise diplomática e análise tipológica de documento de arquivo. São Paulo: Arquivo do Estado e Imprensa Oficial do Estado. Como fazer volume 8.

BLANCO, R. R. (1987) Estudos paleográficos. São Paulo: Laserprint.

CAMPOS, V. S. (1952) Fundações municipais paulistas nos séculos XVIII e XIX. São Paulo: Impres, v. 1.

COSTA, R. F. (2006) Simplificação ou simplicidade da escrita? In: Histórica. Revista on-line do Arquivo do Estado de São Paulo. Edição 15. Outubro de 2006. Disponível em: <http:// www.historica.arquivoestado.sp.gov.br/materias/anteriores/edicao15/materia01/\#topo >. Acessado em 08 de outubro 2007.

CunHA, A. G. da; CAMBRAiA, C. N.; MEgale, H. (2001) A carta de Pero Vaz de Caminha. 2. ed. São Paulo: Humanitas.

FLEXOR, M. H. O. (1990) Abreviaturas. Manuscritos dos séculos XVI ao XIX. 2. ed. São Paulo: Arquivo do Estado.

MEGALE, H. (2001) A demanda do Santo Graal: das origens ao códice português. São Paulo: Ateliê.

. (org.) (2000) Filologia bandeirante. Estudos 1. São Paulo: Humanitas.

MENDES, U. D. (1953) Noções de paleografia. São Paulo: João Bentivegna.

MILLARES CARLO, A. (1929) Paleografía Española. Ensayo de una Historia de la Escritura en España desde el siglo VIII al XVII. Barcelona/Buenos Aires: Labor.

NUNES, E. B. (1981) Abreviaturas paleográficas portuguesas. 3. ed. Lisboa: Faculdade de Letras. NUNES, J. J. (1989) Compêndio de gramática histórica portuguesa. Lisboa: Clássica Editora.

OLIVEIRA, F. de (1975) A gramática da linguagem portuguesa. Introdução, leitura atualizada e notas por Maria Leonor Carvalho Buesco. Lisboa: Imprensa Nacional - Casa da Moeda [1536]. 
SPINA, S. (1977) Introdução à edótica: crítica textual. São Paulo: Cultrix.

VASCONCELOS, J. L. de. (1928) Opúsculos. Coimbra: Imprensa da Universidade, v. 1.

VIANA, A. R. G. (1904) Ortografia Nacional: simplificação e uniformização sistemática das ortografia portuguesas. Lisboa: Livraria Editora Viúva Tavares Cardoso.

VIARO, M. E. (2004) Por trás das palavras: manual de etimologia do português. São Paulo: Globo.

ABSTRACT: This article, which is part of the thesis Formation and expansion of the caipira dialect from Capivari written under the direction of Prof. Dr. Manoel Mourivaldo Santiago Almeida from the Universidade de São Paulo, employs a philological basis to edit 75 official folios written during the 19th century in Capivari in order to prove the fidedignity of the documents and to help the researchers in Philology who study calligraphy and abbreviations. With the aim of helping future studies, this article displays facsimilar reproductions of abbreviations.

KEYWORDS: Folio edition; abbreviations in the 19th century; paleography; philology. 